\title{
Insensitizing controls for a semilinear heat equation with a superlinear nonlinearity
}

\author{
Olivier BODART ${ }^{\mathrm{a}}$, Manuel GONZÁLEZ-BURGOS ${ }^{\mathrm{b}}$, Rosario PÉREZ-GARCÍA ${ }^{\mathrm{b}}$
}

a Laboratoire de Mathématiques Appliquées, UMR CNRS 6620, Université Blaise-Pascal (Clermont-Ferrand 2), 63177 AUBIERE, France

Phone: (33) 4734079 65. Fax: (33) 473407064

E-mail: Olivier.Bodart@math.univ-bpclermont.fr

b Dpto. EDAN, Universidad de Sevilla, Aptdo. 1160, 41080 Sevilla, Spain Phone: (34) 9545579 99, (34) 9545579 96. Fax: (34) 954552898

E-mail: burgos@numer.us.es, rosario@numer.us.es

(Reçu le jour mois année, accepté après révision le jour mois année)

Abstract. This Note is concerned with the existence of insensitizing controls for a semilinear heat equation when we consider nonlinearities that behave superlinearly at infinity. We prove the existence of a control insensitizing the $L^{2}$-norm of the observation of the solution in an open subset $\mathcal{O}$ of the considered domain under appropriate assumptions on the nonlinear term $f(y)$ and the second member $\xi$ of the equation. The proof uses global Carleman estimates, parabolic regularity and a fixed point argument. (C) 2002 Académie des sciences/Éditions scientifiques et médicales Elsevier SAS

Contrôles insensibilisants pour une équation de la chaleur semilinéaire avec non-linéarité superlinéaire

Résumé. $\quad$ Dans cette Note, on étudie l'existence de contrôles insensibilisants pour une équation de la chaleur semi-linéaire, avec des non-linéarités superlinéaires à l'infini. On démontre l'existence de contrôles insensibilisant la norme $L^{2}$ de la solution observée dans un ouvert $\mathcal{O}$ inclus dans le domaine considéré, sous des hypothèses convenables sur la non-linéarité et le second membre de l'équation. La démonstration fait appel à des inégalités de Carleman globales, la régularisation parabolique et un argument de point fixe. (c) 2002 Académie des sciences/Éditions scientifiques et médicales Elsevier SAS

\section{Version française abrégée}

Soit $\Omega \subset \mathbb{R}^{N}, N \geq 1$, un ouvert borné et régulier. Soit $f$ une fonction de classe $C^{1}$ définie sur $\mathbb{R}$. Soient $\omega$ et $\mathcal{O}$ deux ouverts inclus dans $\Omega$. Pour $T>0$ on pose $Q=\Omega \times(0, T)$ et

$\overline{\text { Note présentée par First name NAME }}$

S0764-4442(00)0????-?/FLA

(c) 2002 Académie des sciences/Éditions scientifiques et médicales Elsevier SAS. Tous droits réservés. 1 


\section{O. Bodart, M. González-Burgos, R. Pérez-García}

$\Sigma=\partial \Omega \times(0, T)$. On considère une solution $y=y(x, t ; v, \tau)$ de l'équation $(1)$, où $\xi \in L^{2}(Q)$ et $y_{0} \in L^{2}(\Omega)$ sont données, $\hat{y}_{0} \in L^{2}(\Omega)$ est inconnu avec $\left\|\hat{y}_{0}\right\|_{L^{2}(\Omega)}=1, \tau$ est un petit nombre réel inconnu et $v \in L^{2}(Q)$ est un contrôle à déterminer. On désigne par $1_{\omega}$ la fonction caractéristique de $\omega$.

Soit la fonctionnelle $\phi$ définie par (2), on dit qu'un contrôle $v$ insensibilise $\phi$ si on a (3). Ce problème, posé par J.L. Lions dans [9] fut d'abord étudié dans [1], où les auteurs prouvent l'existence de contrôles approximativement insensibilisants. L. de Teresa a prouvé par ailleurs dans [10], d'une part, qu'il ne peut pas exister de contrôle insensibilisant pour tout $y_{0} \in L^{2}(\Omega)$ quand $\Omega \backslash \bar{\omega} \neq \emptyset$. D'autre part, lorsque $y_{0}=0$ et sous des hypothèses convenables sur $\xi$, elle prouve l'existence de contrôles assurant (3). Dans cette Note, nous étendons ce travail à des non-linéarités plus générales.

L'existence d'un contrôle $v$ satisfaisant à (3) est équivalente à l'existence d'un contrôle $v$ tel que la solution $(y, q)$ de la cascade de systèmes (4) vérifie (5), cf. [9] et [1] pour la démonstration. Le résultat principal de cette Note est le suivant :

THÉORÈme 0.1. - Supposons que $\omega \cap \mathcal{O} \neq \emptyset$ et $y_{0}=0$. Supposons en outre que $f$ est de classe $C^{2}$ telle que $f(0)=0$ et

$$
\lim _{|s| \rightarrow \infty} \frac{f^{\prime}(s)}{\log (1+|s|)}=0 .
$$

Soit $r \in(N+2, \infty)$ donné. Alors, pour tout $\xi \in L^{r}(Q)$ tel que

$$
\iint_{Q} \exp \left(\frac{1}{t^{3}}\right)|\xi|^{2} d x d t<\infty,
$$

il existe un contrôle $v \in L^{r}(Q)$ qui insensibilise la fonctionnelle définie en (2).

La structure de la preuve est basée sur un argument de linéarisation et point fixe, l'étude fine du cas linéaire en étant le point clé comme dans la plupart des démonstrations de ce type (voir [6], $[11], \ldots)$.

On considère le problème de contrôle linéaire (8)-(9). Soient $B_{0}$ et $B$ deux ouverts tels que $B_{0} \subset \subset B \subset \omega \cap \mathcal{O}$. Dans un premier temps, on construit un contrôle $\hat{v} \in L^{2}(Q)$, à support dans $\bar{B}_{0} \times[0, T]$, grâce à une inégalité d'observabilité mixte. Cette inégalité fut d'abord donnée dans [10], ainsi que la construction du contrôle qui en découle. Dans [2], elle est étendue à des systèmes plus généraux, et la dépendance des constantes vis à vis des potentiels est explicitée. On obtient ainsi une estimation de la norme de $\hat{v}$ en fonction des paramètres $a$ et $b$. On note $(\hat{y}, \hat{q})$ la solution de (8)-(9) associée au contrôle $\hat{v}$.

Ensuite, on considère une fonction telle que $\theta \equiv 1$ dans un voisinage de $B_{0}$. On pose alors

$$
q=(1-\theta) \hat{q} \quad \text { and } \quad y=(1-\theta) \hat{y}+2 \nabla \theta \cdot \nabla \hat{q}+(\Delta \theta) \hat{q} .
$$

Le couple $(y, q)$ vérifie $(8),(9)$ avec le contrôle

$$
v=-\theta \xi+2 \nabla \theta \cdot \nabla \hat{y}+(\Delta \theta) \hat{y}+\left(\partial_{t}-\Delta+a\right)[2 \nabla \theta \cdot \nabla \hat{q}+(\Delta \theta) \hat{q}] .
$$

Ce contrôle est à support dans $\bar{B} \times[0, T]$. En utilisant les propriétés de régularisation de l'équation de la chaleur, sous des hypothèses convenables sur $a$ et $b$, on montre que $v \in L^{r}(Q)$ et satisfait l'estimation (10).

La régularité de $v$ permet d'obtenir des estimations sur $y$ dans un espace de Hölder dont l'injection est compacte dans $Z=C^{0}(\bar{Q}) \cap L^{r}\left(0, T ; W^{1, r}(\Omega)\right)$. On pose

$$
g(s)=\left\{\begin{array}{cl}
\frac{f(s)}{s} & \text { if } s \neq 0 \\
f^{\prime}(0) & \text { if } s=0
\end{array}\right.
$$


Pour $z \in Z$, on considère le système linéaire (8) avec les potentiels $a=a_{z}=g(z) \in L^{\infty}(Q)$, $b=b_{z}=f^{\prime}(z) \in L^{\infty}(Q) \cap L^{r}\left(0, T ; W^{1, r}(\Omega)\right)$. D'après ce qui précède, il existe $v_{z} \in L^{r}(Q)$ tel que la solution correspondante $\left(y_{z}, q_{z}\right)$ de (8) vérifie (9) et $y_{z}, q_{z} \in Z$. On conclut la preuve en appliquant le théorème du point fixe de Kakutani à l'application $z \mapsto y_{z}$. Les détails sont donnés dans [3].

\section{Statement of the problem}

Let $\Omega \subset \mathbb{R}^{N}, N \geq 1$, be a regular bounded open set. Let $f$ be a $C^{1}$ function defined on $\mathbb{R}$. Let $\omega$ and $\mathcal{O}$ be two open subsets of $\Omega$. For $T>0$ we denote $Q=\Omega \times(0, T)$ and $\Sigma=\partial \Omega \times(0, T)$. We consider the semilinear heat equation:

$$
\begin{cases}\partial_{t} y-\Delta y+f(y)=\xi+v 1_{\omega} & \text { in } Q, \\ y=0 \text { on } \Sigma, \quad y(0)=y_{0}+\tau \hat{y}_{0} & \text { in } \Omega,\end{cases}
$$

where $\xi \in L^{2}(Q)$ and $y_{0} \in L^{2}(\Omega)$ are given, $\hat{y}_{0} \in L^{2}(\Omega)$ is unknown with $\left\|\hat{y}_{0}\right\|_{L^{2}(\Omega)}=1, \tau$ is a small unknown real number and $v \in L^{2}(Q)$ is a control function to be determined. Here $1_{\omega}$ is the characteristic function of $\omega$.

Let us define

$$
\phi(y)=\frac{1}{2} \iint_{\mathcal{O} \times(0, T)}|y(x, t ; \tau, v)|^{2} d x d t,
$$

$y=y(\cdot, \cdot ; \tau, v)$ being a solution of (1) associated to $\tau$ and $v$. A control function $v$ is said to insensitize $\phi$ if

$$
\left.\frac{\partial \phi(y(\cdot, \cdot ; \tau, v))}{\partial \tau}\right|_{\tau=0}=0, \quad \forall \hat{y}_{0} \in L^{2}(\Omega) \text { with }\left\|\hat{y}_{0}\right\|_{L^{2}(\Omega)}=1 .
$$

This problem, addressed by J.L. Lions in [9], has been studied for globally Lipschitz-continuous nonlinearities. First, in [1] the authors proved the existence of so-called approximately insensitizing controls for unknown data both in the initial and boundary conditions. In [10] it was proved, on the one hand, that we cannot expect the existence of insensitizing controls for every $y_{0} \in L^{2}(\Omega)$ when $\Omega \backslash \bar{\omega} \neq \emptyset$. On the other hand, for $y_{0}=0$ and suitable assumptions on $\xi$, L. de Teresa proved the existence of controls such that (3) holds. In this note we extend this last result to more general nonlinearities.

It is shown in [9] and [1] that the existence of a control $v$ satisfying (3) is equivalent to the existence of a control $v$ such that the solution $(y, q)$ of

$$
\left\{\begin{array}{l}
\partial_{t} y-\Delta y+f(y)=\xi+v 1_{\omega}, \quad-\partial_{t} q-\Delta q+f^{\prime}(y) q=y 1_{\mathcal{O}} \quad \text { in } Q \\
y=0, \quad q=0 \quad \text { on } \Sigma, \quad y(x, 0)=y_{0}(x), \quad q(x, T)=0 \quad \text { in } \Omega
\end{array}\right.
$$

verifies

$$
q(x, 0)=0 \text { in } \Omega .
$$

The main result in this note is the following

Theorem 1.1. - Assume that $\omega \cap \mathcal{O} \neq \emptyset$ and $y_{0}=0$. Let $f$ be a $C^{2}$ function verifying $f(0)=0$ and

$$
\lim _{|s| \rightarrow \infty} \frac{f^{\prime}(s)}{\log (1+|s|)}=0 .
$$




\section{O. Bodart, M. González-Burgos, R. Pérez-García}

Let $r \in(N+2, \infty)$ be given. Then, for any $\xi \in L^{r}(Q)$ such that

$$
\iint_{Q} \exp \left(\frac{1}{t^{3}}\right)|\xi|^{2} d x d t<\infty
$$

there exists a control function $v \in L^{r}(Q)$ insensitizing the functional given by (2).

As usual in controllability problems for nonlinear equations, we first prove a controllability result for a linearized version of (4). We then apply a fixed point argument to treat the general case. The structure of the proof is quite general (for other controllability results proved in a similar way, see $[6],[11], \ldots)$. We sketch here these two main steps and the details will be given in [3].

Remark 1. - Hypothesis (6) is fulfilled by certain superlinear nonlinearities $f$ such as

$$
|f(s)|=\left|p_{1}(s)\right| \log ^{\alpha}\left(1+\left|p_{2}(s)\right|\right) \quad \text { for all }|s| \geq s_{0}>0,
$$

with $\alpha \in[0,1)$, where $p_{1}$ and $p_{2}$ are first order real polynomial functions.

In view of known null-controllability results for a semilinear heat equation with homogeneous Dirichlet boundary conditions (see Theorem 1.2 in [6]), one could think of extending Theorem 1.1 to nonlinearities $f$ with growth at infinity of order $|s| \log ^{\alpha}(1+|s|)$ with $1 \leq \alpha<3 / 2$. Notice that, in this case, in the absence of control, blow-up phenomena occur. The idea in [6] is to control the system to zero in a short time to prevent the solution from blowing-up and, the only right hand side term being the control, setting $v \equiv 0$ for the rest of the time interval. However, this strategy fails here since, in insensitivity problems, both initial and final times are fixed and, in addition, there is a right hand side term $\xi$ in the equation. The problem then remains opened for such nonlinearities.

When $f \equiv 0$ and $\mathcal{O}=\Omega$, a positive result on the existence of insensitizing controls is proved for a small class of initial data (see Lemma 2 in [10]). The proof of this result is strongly based on properties of the semigroup of the classical heat equation. Even in the case of a linear heat equation with an $L^{\infty}$ potencial, giving sufficient conditions on the initial data $y_{0}$ in order to insensitize (2) is an open problem.

\section{The linear case}

Let us consider the following linear controllability problem:

$$
\begin{aligned}
& \left\{\begin{array}{l}
\partial_{t} y-\Delta y+a y=\xi+v 1_{\omega}, \quad-\partial_{t} q-\Delta q+b q=y 1_{\mathcal{O}} \quad \text { in } Q \\
y=0, \quad q=0 \quad \text { on } \Sigma, \quad y(x, 0)=0, \quad q(x, T)=0 \quad \text { in } \Omega .
\end{array}\right. \\
& q(0)=0 \text { in } L^{2}(\Omega),
\end{aligned}
$$

where $a, b \in L^{\infty}(Q)$ and $\xi, \omega, \mathcal{O}$ are given as in Theorem 1.1.

First, using an adequate observability inequality, we construct controls in $L^{2}(Q)$. Then, due to the regularizing properties of the heat equation, we exhibit more regular controls. This will be essential in the proof of Theorem 1.1 since $f$ behaves superlinearly at infinity. We actually seek a control supported in $\omega \cap \mathcal{O} \neq \emptyset$. Let then $B_{0}$ and $B$ be two open sets such that $B_{0} \subset \subset B \subset \omega \cap \mathcal{O}$. The following result holds:

Proposition 2.1. - Let $\xi$ be as in Theorem 1.1. Then, there exists a control $\hat{v} \in L^{2}(Q)$ such that supp $\hat{v} \subset \bar{B}_{0} \times[0, T]$ and the corresponding solution $(\hat{y}, \hat{q})$ of (8) satisfies $(9)$. Moreover, $\hat{v}$ can be chosen in such a way that

$$
\|\hat{v}\|_{L^{2}(Q)}^{2} \leq \exp \left[C H\left(T,\|a\|_{\infty},\|b\|_{\infty}\right)\right] \iint_{Q} \exp \left[C \frac{M\left(T,\|a\|_{\infty},\|b\|_{\infty}\right)}{t}\right]|\xi|^{2} d x d t
$$


with

$$
\begin{gathered}
M\left(T,\|a\|_{\infty},\|b\|_{\infty}\right)=1+T+T\|a\|_{\infty}^{2 / 3}+T\|b\|_{\infty}^{2 / 3}+T\|a-b\|_{\infty}^{1 / 2} \\
H\left(T,\|a\|_{\infty},\|b\|_{\infty}\right)=1+\frac{1}{T}+T+T\|a\|_{\infty}+\|a\|_{\infty}^{2 / 3}+T\|b\|_{\infty}+\|b\|_{\infty}^{2 / 3}+\|a-b\|_{\infty}^{1 / 2}
\end{gathered}
$$

$C$ being a positive constant only depending on $\Omega, \omega$ and $\mathcal{O}$.

A similar result was proved in [10] as a consequence of a global Carleman inequality for the linear heat equation (cf. [7]). In [2], the result is extended to the linear heat equation involving first order terms and one gives the explicit dependence of $M$ and $H$ on $T,\|a\|_{\infty}$ and $\|b\|_{\infty}$. This is crucial to prove the existence of insensitizing controls in the nonlinear case when $f$ is not a globally Lipschitz-continuous function.

From $(\hat{y}, \hat{q})$ we now build a new control $v \in L^{r}(Q)$ with support in $\bar{B} \times[0, T], r$ being as in Theorem 1.1. For this, let $\theta \in \mathcal{D}(B)$ be a function such that $\theta \equiv 1$ in a neighbourhood of $B_{0}$. We set

$$
q=(1-\theta) \hat{q} \quad \text { and } \quad y=(1-\theta) \hat{y}+2 \nabla \theta \cdot \nabla \hat{q}+(\Delta \theta) \hat{q} .
$$

Then, $(y, q)$ solves (8), (9) for the control

$$
v=-\theta \xi+2 \nabla \theta \cdot \nabla \hat{y}+(\Delta \theta) \hat{y}+\left(\partial_{t}-\Delta+a\right)[2 \nabla \theta \cdot \nabla \hat{q}+(\Delta \theta) \hat{q}] .
$$

In the sequel $C$ will stand for a generic positive constant depending on $\Omega, \omega, \mathcal{O}$ and $T$, which value can change from line to line. One has

Proposition 2.2. -

1. For $a, b \in L^{\infty}(Q)$ we have $y, q \in C^{0}(\bar{Q}) \cap L^{r}\left(0, T ; W^{1, r}(\Omega)\right)$ and

$$
\|y\|_{C^{0}(\bar{Q}) \cap L^{r}\left(W^{1, r}\right)}+\|q\|_{C^{0}(\bar{Q}) \cap L^{r}\left(W^{1, r}\right)} \leq \exp \left[C\left(1+\|a\|_{\infty}+\|b\|_{\infty}\right)\right]\left(\|\xi\|_{L^{r}(Q)}+\|\hat{v}\|_{L^{2}(Q)}\right) .
$$

2. Suppose in addition that $b \in L^{r}\left(0, T ; W^{1, r}(\Omega)\right)$. Then $v \in L^{r}(Q)$, supp $v \subset \bar{B} \times[0, T]$ and

$$
\|v\|_{L^{r}(Q)} \leq \exp \left[C\left(1+\|a\|_{\infty}+\|b\|_{\infty}\right)\right]\left(1+\|\nabla b\|_{L^{r}(Q)^{N}}\right)\left(\|\xi\|_{L^{r}(Q)}+\|\hat{v}\|_{L^{2}(Q)}\right) .
$$

The proof of this result can be found in [3] and combines the regularizing effect and properties of the semigroup generated by the heat equation with Dirichlet boundary conditions.

Remark 2. - In the case of the heat equation, the previous technique provides a new method of construction of more regular controls from controls in $L^{2}(Q)$. This allows to prove both null controllability and insensitizing local results for the heat equation with nonlinear Fourier boundary conditions (see $[5,4])$.

\section{Idea of the proof of Theorem 1.1}

Let $f, \xi$ be as in Theorem 1.1. Let us define

$$
g(s)=\left\{\begin{array}{cc}
\frac{f(s)}{s} & \text { if } s \neq 0 \\
f^{\prime}(0) & \text { if } s=0 .
\end{array}\right.
$$

Let us set

$$
Z=C^{0}(\bar{Q}) \cap L^{r}\left(0, T ; W^{1, r}(\Omega)\right) .
$$

For any $z \in \bar{B}(0 ; R) \subset Z, R>0$ being positive, we consider the linear system (8) with potentials $a=a_{z}=g(z) \in L^{\infty}(Q), b=b_{z}=f^{\prime}(z) \in L^{\infty}(Q) \cap L^{r}\left(0, T ; W^{1, r}(\Omega)\right)$. In view of Propositions 2.1 


\section{O. Bodart, M. González-Burgos, R. Pérez-García}

and 2.2 and the assumptions on $\xi$, we infer that there exists $v_{z} \in L^{r}(Q)$ such that the corresponding solution $\left(y_{z}, q_{z}\right)$ of $(8)$ satisfies $y_{z}, q_{z} \in Z,(9)$ and

$$
\begin{gathered}
\left\|y_{z}\right\|_{Z} \leq \exp \left[C\left(1+\|g(z)\|_{\infty}+\left\|f^{\prime}(z)\right\|_{\infty}\right)\right]\left(\|\xi\|_{L^{r}(Q)}+\left\|\exp \left(\frac{1}{2 t^{3}}\right) \xi\right\|_{L^{2}(Q)}\right) \\
\left\|v_{z}\right\|_{L^{r}(Q)} \leq C(\Omega, \omega, \mathcal{O}, T, R)\left(\|\xi\|_{L^{r}(Q)}+\left\|\exp \left(\frac{1}{2 t^{3}}\right) \xi\right\|_{L^{2}(Q)}\right) .
\end{gathered}
$$

Let us define $\mathcal{A}: \bar{B}(0 ; R) \subset Z \longmapsto L^{r}(Q)$ and $\Lambda: \bar{B}(0 ; R) \subset Z \longmapsto Z$ as

$$
\begin{gathered}
\mathcal{A}(z)=\left\{v \in L^{r}(Q):(y, q) \text { satisfies }(8) \text { for } a_{z}, b_{z},(9), v \text { verifying }(12)\right\}, \\
\Lambda(z)=\left\{y \in Z:(y, q) \text { solution of }(8) \text { for } a_{z}, b_{z} \text { with } v \in \mathcal{A}(z), y \text { satisfying }(11)\right\} .
\end{gathered}
$$

For fixed $z \in \bar{B}(0 ; R), \Lambda(z)$ is a non empty convex closed set in $Z$. In fact, in view of Theorem 9.1 p. 341 in [8], its Corollary and $(12), \Lambda(z)$ is uniformly bounded in the Hölder space $H^{1+\beta,(1+\beta) / 2}(\bar{Q})$ with $\beta=1-(N+2) / r$. The imbedding of $H^{1+\beta,(1+\beta) / 2}(\bar{Q})$ being compact in $Z$, there exists a compact set $K \subset Z$ such that $\Lambda(z) \subset K$ for every $z \in \bar{B}(0 ; R)$. Due to (6), (11) we infer the existence of $R>0$ large enough such that $\Lambda(\bar{B}(0 ; R)) \subset \bar{B}(0 ; R)$. Finally, one can prove that $\Lambda$ is an upper hemicontinuous multivalued mapping. We can then apply Kakutani's fixed point theorem and conclude that there exists $y \in Z$ such that $y \in \Lambda(y)$. This ends the proof of Theorem 1.1.

\section{References}

[1] O. Bodart, C. Fabre, Controls insensitizing the norm of the solution of a semilinear heat equation, J. Math. Anal. Appl. 195 (3) (1995) 658-683.

[2] O. Bodart, M. González-Burgos, R. Pérez-García, Insensitizing controls for a heat equation with a nonlinear term involving the state and the gradient (in preparation).

[3] O. Bodart, M. González-Burgos, R. Pérez-García, Existence of insensitizing controls for a semilinear heat equation with a superlinear nonlinearity (in preparation).

[4] O. Bodart, M. González-Burgos, R. Pérez-García, A local result on insensitizing controls for the heat equation with nonlinear boundary Fourier conditions (in preparation)

[5] A. Doubova, E. Fernández-Cara, M. González-Burgos, On the controllability of the heat equation with nonlinear boundary Fourier conditions (in preparation)

[6] E. Fernández-Cara, E. Zuazua, Null and approximate controllability for weakly blowing up semilinear heat equations, Ann. Inst. H. Poincaré Anal. Non Linéaire 17 (5) (2000) 583-616.

[7] A. Fursikov, O.Yu. Imanuvilov, Controllability of Evolution Equations, Lecture Notes Series \#34, Seoul National University, Seoul, 1996.

[8] O.A. Ladyzenskaya, V.A. Solonnikov, N.N. Uraltzeva, Linear and Quasilinear Equations of Parabolic Type, Nauka, Moskow, 1967.

[9] J.L. Lions, Quelques notions dans l'analyse et le contrôle de systèmes à données incomplètes, Proceedings of the XIth Congress on Differential Equations and Applications/First Congress on Applied Mathematics, University of Málaga, Málaga, 1990, 43-54.

[10] L. De Teresa, Insensitizing controls for a semilinear heat equation, Commun. Partial Differential Equations 25 (1\&2) (2000) 39-72.

[11] E. Zuazua, Exact boundary controllability for the semilinear wave equation, in: Brezis H., Lions J.L. (Eds.), NonLinear Partial Differential Equations and their Applications Vol. X, Pitman, 1991, 357-391. 